\title{
Mobile X-rays are highly valuable for critically ill COVID patients
}

\author{
Gang $\mathrm{Wu}^{1} \cdot$ Xiaoming $\mathrm{Li}^{1}$ (D)
}

Received: 31 March 2020 /Revised: 7 April 2020 / Accepted: 24 April 2020 / Published online: 13 May 2020

(C) European Society of Radiology 2020

Dear Editor,

The diagnostic value of CT for SARS-CoV-2 infection has been widely reported $[1,2]$. However, performing $\mathrm{CT}$ is very difficult in critically ill patients in ICU, whereas mobile Xrays serve as an alternative. From Feb. 20 to Mar. 20, 542 mobile chest X-rays were performed in 229 COVID patients who were under assisted ventilation. Table 1 summarizes the main characteristics of the cohort.

Among the 229, a significant underlying disease existed in $165(72 \%)$ : hypertension in $31 \%$, chronic heart disease in $13 \%$, chronic pulmonary disease in $12 \%$, and malignancy in $11 \%$. As of Mar. 30, $95(41 \%)$ of 229 patients had died, and 134 (59\%) survived. Among them, 58 were discharged and 76 still in hospital.

Table 1 shows the number of X-rays performed per patient. There were 229 initial examination and 313 were follow-ups. The image quality was diagnostic for all these $\mathrm{X}$-rays.

Extensive pneumonia was seen on the initial X-rays in all patients. Other findings were pneumothorax (7\%) and pleural effusion (23\%). Subcutaneous emphysema, hiatal hernia, and intrapulmonary cavity were rare findings (Table 1). All patients with pneumothorax died. Compared to previous X-rays, increase of pneumonia was seen in 119 (38\%) of 313 followup X-rays, while no changes were seen in 57 (18\%), and improvement in 137 (44\%).

Among non-survivors $(n=95), 61$ patients did not have follow-up X-rays and died 1 4 days after the initial examination; 34 patients had 55 follow-up X-rays. Pneumonia progress was seen in $53(96 \%)$ out of 55 follow-up X-rays when compared to previous radiograph (Fig. 1).

Please note that due to the time sensitive nature of the work presented in this article, standard peer review has been bypassed to ensure rapid publication. The article has been directly assessed by the Editor-in-Chief.

Xiaoming Li

lilyboston2002@qq.com

1 Department of Radiology, Tongji Hospital of Tongji Medical College of Huazhong University of Science and Technology, Wuhan, China
Among survivors $(n=134), 111$ patients had 258 followup X-rays. Pneumonia progress was seen in 66/258 radiographs (26\%) of follow-up X-rays in 37 survivors; however, improvement was seen later.

We found mobile $\mathrm{X}$-rays provided adequate image quality for diagnosing pneumonia, at least in this population of severely ill patients. Although obviously less informative than CT, X-rays can nevertheless detect serious complications like pneumothorax or lung cavitation, and estimate the evolution of pneumonia. Therefore, it is a simple but reliable alternative in critically ill SARS-CoV-2 patients who could not undergo chest $\mathrm{CT}$.

Funding information This work received financial support from the National Natural Science of Foundation of China, No. 81801663, 31630025 , and 81571643 .

\section{Compliance with ethical standards}

Guarantor The scientific guarantor of this publication is Wenzhen Zhu.

Conflict of interest The authors declare that they have no conflict of interest.

Statistics and biometry No complex statistical methods were necessary for this paper.

Informed consent Not applicable.

Ethical approval Institutional Review Board approval was not required because this publication is a Letter to the Editor.

Methodology

- Not applicable 
Table 1 The main characteristics of 229 patients with SARS-CoV-2 pneumonia with mobile X-rays

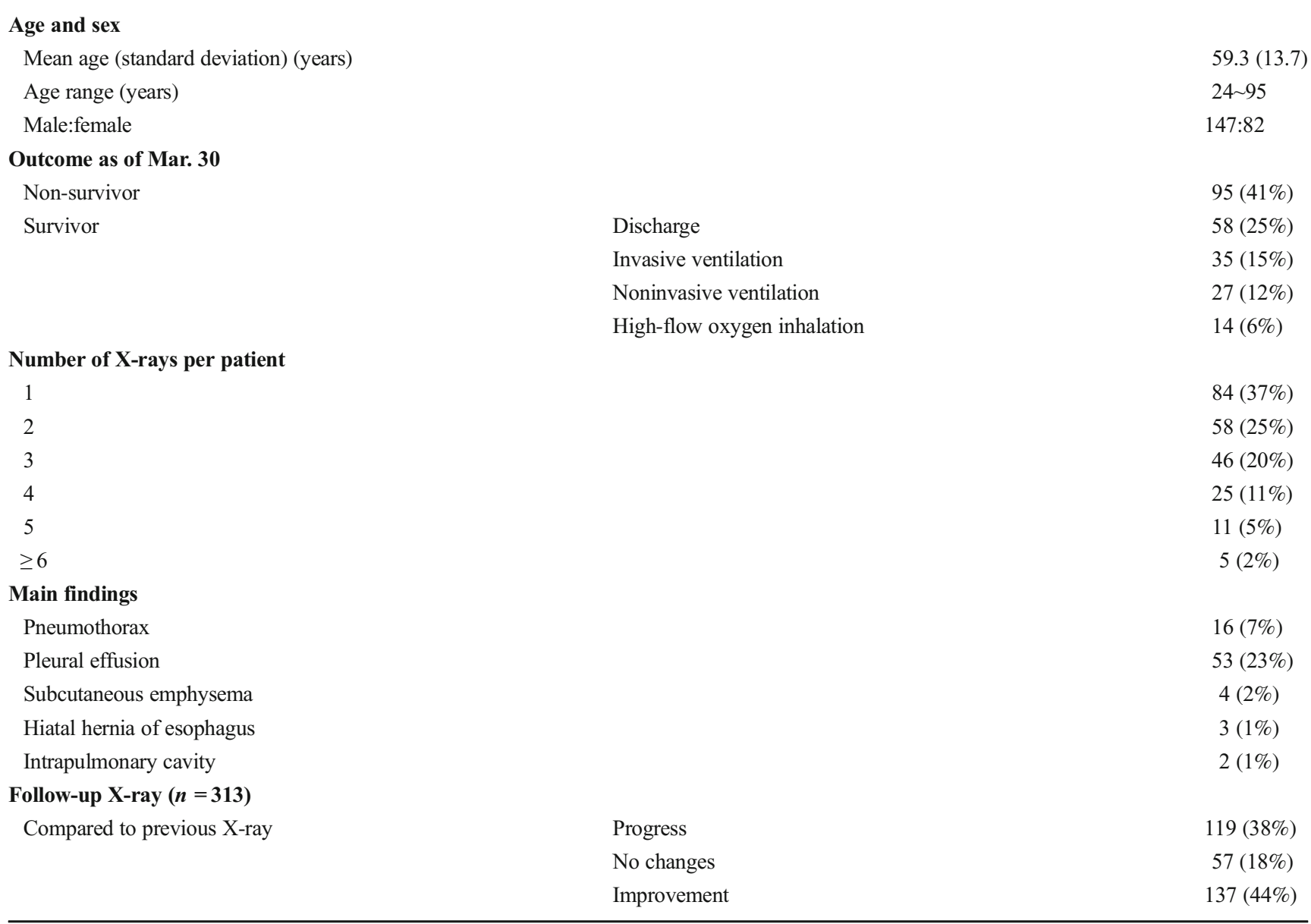

$S A R S-C o V-2=$ severe acute respiratory syndrome coronavirus 2

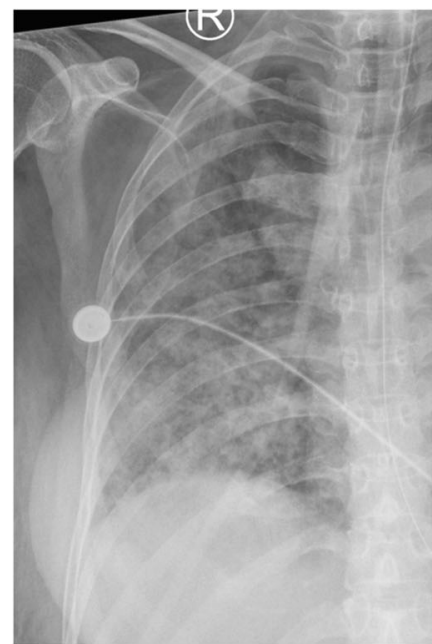

2.21

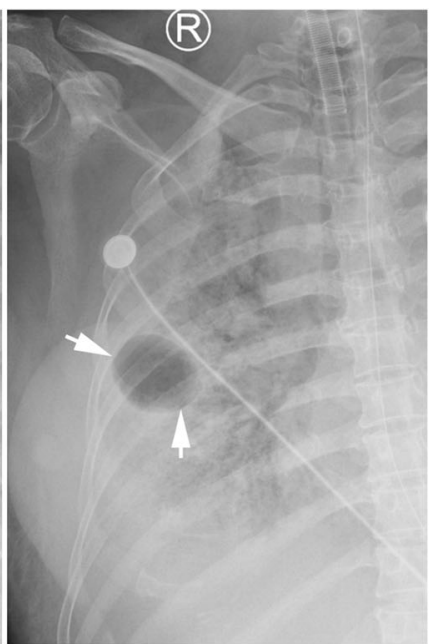

2.24

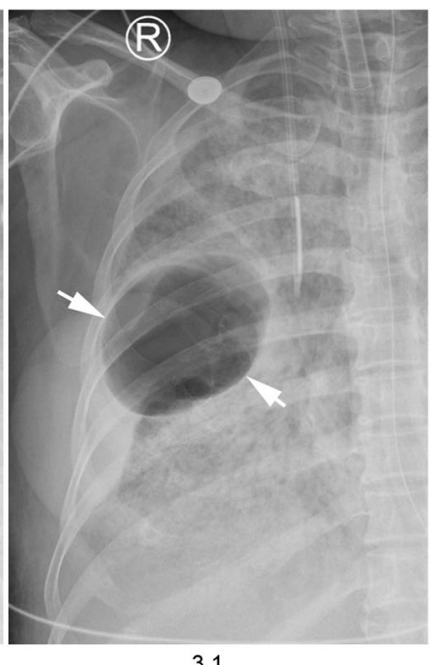

3.1
Fig. 1 A 57-year-old female with laboratory-confirmed SARS-CoV-2 pneumonia had dyspnea one week after onset of symptoms of fever and diarrhea. Under high-flow oxygen inhalation, her pulse oxygen saturation (SPO2) was only $60 \%$; thus, invasive ventilation was used with fraction of inspired oxygen $50-70 \%$ to maintain SPO2 around 95\%. On Feb. 21, she underwent the first mobile X-ray, which indicated extensive pneumonia. Compared with initial X-ray, significant progress could be seen in the follow-up X-ray on Feb. 24, which was a typical "white lung." A new intrapulmonary cavity was revealed by the second X-ray, and was significantly larger in the third X-ray on Mar. 1. The patient died of respiratory failure four days after the last X-ray 


\section{References}

1. Ye Z, Zhang Y, Wang Y, Huang Z, Song B (2020) Chest CT manifestations of new coronavirus disease 2019 (COVID-19): a pictorial review. Eur Radiol. https://doi.org/10.1007/s00330-020-06801-0
2. Kim H (2020) Outbreak of novel coronavirus (COVID-19): what is the role of radiologists? Eur Radiol. https://doi.org/10.1007/s00330020-06748-2

Publisher's note Springer Nature remains neutral with regard to jurisdictional claims in published maps and institutional affiliations. 